\title{
Toward a Comprehensive Global Emission Inventory of C4-C10 Perfluoroalkanesulfonic Acids (PFSAs) and Related Precursors: Focus on the Life Cycle of C6- and C10-Based Products
}

\section{Journal Article}

\section{Author(s):}

Boucher, Justin M.; Cousins, lan T.; Scheringer, Martin; Hungerbühler, Konrad; Wang, Zhanyun

Publication date:

2018

Permanent link:

https://doi.org/10.3929/ethz-b-000308867

Rights / license:

$\underline{\text { In Copyright - Non-Commercial Use Permitted }}$

Originally published in:

Environmental Science \& Technology Letters 6(1), https://doi.org/10.1021/acs.estlett.8b00531 


\title{
Toward a Comprehensive Global Emission Inventory of $\mathrm{C}_{4}-\mathrm{C}_{10}$ Perfluoroalkanesulfonic Acids (PFSAs) and Related Precursors: Focus on the Life Cycle of $\mathrm{C}_{6}$ - and $\mathrm{C}_{10}$-Based Products
}

\author{
Justin M. Boucher, ${ }^{* \dagger \odot}$ Ian T. Cousins, ${ }^{\ddagger}{ }^{\dagger}$ Martin Scheringer, ${ }^{\dagger, \S, \| \odot ~ K o n r a d ~ H u n g e r b u ̈ h l e r, ~}$
} and Zhanyun Wang $*,+\perp$ (

\footnotetext{
${ }^{\dagger}$ Safety and Environmental Technology Group, Institute for Chemical and Bioengineering, ETH Zurich, 8093 Zurich, Switzerland

${ }^{*}$ Department of Environmental Science and Analytical Chemistry (ACES), Stockholm University, 10691 Stockholm, Sweden

${ }^{\S}$ Institute of Biogeochemistry and Pollutant Dynamics, ETH Zurich, 8092 Zurich, Switzerland

${ }^{\|}$RECETOX, Masaryk University, 62500 Brno, Czech Republic

${ }^{\perp}$ Chair of Ecological Systems Design, Institute of Environmental Engineering, ETH Zurich, 8093 Zurich, Switzerland
}

\author{
Supporting Information
}

ABSTRACT: A first global emission inventory of $\mathrm{C}_{4}-\mathrm{C}_{10}$ perfluoroalkanesulfonic acids (PFSAs) released during the life cycle of perfluorohexanesulfonyl fluoride (PHxSF)- and perfluorodecanesulfonyl fluoride (PDSF)-based products is presented. This study complements previous research on emissions of PFSAs that focused largely on the life cycle of perfluorooctanesulfonyl fluoride (POSF) and its derivatives. It reviews and integrates existing information about the life cycle of PHxSF, PDSF, and their derivatives; the limited data available in the public domain point to potentially significant global production, uses, and releases of these substances.

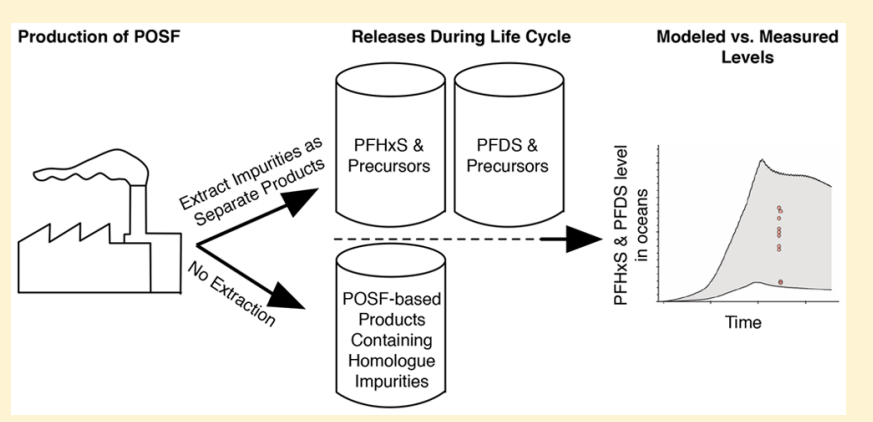
(PFDS) are estimated to be 120-1022 and 38-378 metric tons, respectively. With the new emission estimates as inputs in a global multimedia environmental fate model (CliMoChem), the model-derived environmental concentrations well capture the reported field concentrations, providing strong support for the plausibility of the developed emission inventories. The results highlight the ongoing environmental exposure to these substances and the need for more detailed data in the public domain about their production levels and uses.

\section{INTRODUCTION}

Substantial efforts have been made in recent years to understand the emissions of perfluoroalkanesulfonic acids (PFSAs) and their precursors into the environment and their subsequent fates. A strong focus has been on the life cycle of intentionally produced perfluorooctanesulfonate (PFOS), its parent compound perfluorooctanesulfonyl fluoride (POSF), and related precursors. ${ }^{1-5}$ In contrast, much less is understood about the global production, use, and releases of the six-carbon chain $\left(\mathrm{C}_{6}\right)$ homologue, perfluorohexanesulfonyl fluoride (PHxSF), the 10-carbon chain $\left(\mathrm{C}_{10}\right)$ homologue, perfluorodecanesulfonyl fluoride (PDSF), and their derivatives, including perfluorohexanesulfonic acid ( $\mathrm{PFHxS}$ ) and perfluorodecanesulfonic acid (PFDS).

Although publicly available information is limited, there are indications that the historical and/or ongoing global production, use, and release may have resulted in substantial environmental and human exposure to PFHxS, PFDS, and their precursors: (1) The thermal and chemical stability, in addition to the hydro- and oleophobicity, of the perfluoroalkyl moiety $\left(\mathrm{C}_{n} \mathrm{~F}_{2 n+1^{-}}\right)$allows PFHxS, PFDS, and their precursors to be used as surfactants and/or surface protectors in a wide range of applications. Reported examples for uses of $\mathrm{PFHxS}$ and its precursors include the following: as additives in aqueous firefighting foams (AFFFs), ${ }^{6-9}$ metal plating, ${ }^{10-14}$ textiles and upholstery, ${ }^{15,16}$ polishing/cleaning agents, ${ }^{17,18}$ and impregnation/proofing agents. ${ }^{19}$ For details about individual uses, see the risk profile recently developed under the Stockholm Convention. ${ }^{20}$ PFDS is reported to have been used as a wetting agent in floor polishes. ${ }^{21}$ (2) Historically, 3M produced these substances on a large scale until 2002, with reported production volumes of approximately 228 metric tons (t) of PHxSF and $11 \mathrm{t}$ of PDSF in the United States in $1997 .^{22}$ (3) Recently, some manufacturers in China and Italy have reportedly produced $\mathrm{PFHxS}$ and its precursors, ${ }^{23-25}$ poten-

Received: October 5, 2018

Revised: November 9, 2018

Accepted: November 13, 2018

Published: November 13, 2018 
tially as replacements for PFOS and its precursors. (4) PFHxS and PFDS are non-naturally occurring substances that have been frequently detected at measurable concentrations in various matrices, including ocean surface water (presented here and in Section S4 of the Supporting Information), drinking water supplies, ${ }^{26}$ blood serum of firefighters, ${ }^{27,28}$ and household dust. ${ }^{29}$ Many studies have further quantified both human and environmental exposure to PFHxS and PFDS globally, and these have recently been aggregated and analyzed for significant time trends. ${ }^{30}$

PFHxS, its salts, and related compounds have been nominated to the Stockholm Convention, ${ }^{31}$ and in September 2018, it was found ${ }^{32}$ that "these substances are likely, as a result of their long range environmental transport, to lead to significant adverse human health and environmental effects such that global action is warranted". PFHxS and its salts were also recently added to the European REACH Candidate List of Substances of Very High Concern (SVHC) for their very persistent and very bioaccumulative ( $\mathrm{vPvB}$ ) properties. $^{33}$

To support the ongoing evaluation process under the Stockholm Convention and to help to improve the understanding of the overall magnitude of human and environmental exposure to these chemicals over time, this study presents global emission inventories of $\mathrm{C}_{4}-\mathrm{C}_{10}$ PFSAs and their precursors throughout the life cycle of $\mathrm{PHxSF}, \mathrm{PDSF}$, and their derivatives.

\section{MATERIALS AND METHODS}

This study builds on our earlier study of POSF and its derivatives $^{3}$ and used the same methodology to develop and validate the emission inventories. In brief, the inventories were calculated by integrating information about the production, use, and disposal of relevant chemicals collected and extrapolated from publicly accessible information with emission factors of respective life stages estimated in our earlier study ${ }^{3}$ (Figures S2-S4 offer a schematic overview of the calculations in the Supporting Information). The inventories were then used as inputs for the global mass-balance environmental fate model, CliMoChem, to estimate environmental concentrations, which were then compared to field measurements. Given the limited availability of data and uncertainties in the parameters involved, ranges of plausible values were implemented in the inventory calculations whenever possible, which resulted in ranges of plausible emission levels and estimated environmental concentrations. Here, the fundamental aspects of the methodology, in particular modifications in comparison to our previous study, $^{3}$ are highlighted. Details, including an uncertainty analysis, are provided in the Supporting Information.

Quantification of Production Volumes. Compared to POSF and its derivatives, only limited quantitative information is available for the production of PHxSF, PDSF, and their derivatives. Theoretically, PHxSF and PDSF may be either intentionally produced by electrochemical fluorination (ECF) of their nonfluorinated analogues ${ }^{34}$ or unintentionally produced as byproducts during production of their homologues by ECF (e.g., POSF). ${ }^{35-37}$

Two important hypotheses guided the inventory development. First, we hypothesized that, apart from currently not quantifiable intentional production of $\mathrm{PHxSF}$ and its derivatives in China and Italy, ${ }^{23,24}$ all of the other PHxSF and PDSF that has existed on the market originated as unintentional impurities during production of POSF. This is based on the following considerations. (1) Production of PHxSF, POSF, and PDSF competes for the same production facilities. (2) The performance of PFHxS and its precursors is likely considerably lower than that of PFOS and its precursors. $^{38,39}$ (3) Although the performance of PFDS and its precursors is likely similar to that of PFOS and its precursors, $^{39}$ the production yields of PDSF are likely substantially lower than those of POSF. ${ }^{40}$ Second, we further hypothesized that prior to 3M's phase-out of POSF-based products in 2000-2002, ${ }^{22}$ manufacturers in the United States, Western Europe, and Japan [collectively here termed country group 1 (CG1)] made the best use of the unintentionally produced PHxSF and PDSF impurities to maximize resource efficiency and profits from the same reaction process. We hypothesized that this PHxSF and PDSF were extracted from the produced POSF (e.g., using distillation) and converted into products that are mentioned in the Introduction. This is supported by earlier $3 \mathrm{M}$ documentation. ${ }^{37,41}$ In contrast, manufacturers in China were hypothesized to not have extracted unintentionally produced PHxSF and PDSF impurities from their POSF production. This is based on high levels of PFHxS found in three commercial PFOS products manufactured in China. ${ }^{42}$

Past and ongoing intentional production of PHxSF, PDSF, and their derivatives in China and Italy ${ }^{23,24}$ cannot be quantified and is not included here because no public information about their production volumes could be found. However, these volumes are expected to be much smaller than the sum of historical and ongoing unintentional production as impurities and are thus expected to have a minimum impact on the overall results and conclusions presented. This is based on the following considerations. (1) No PHxSF or its derivatives were identified to have been registered under the European chemicals regulation (REACH) as of September 2018; i.e., any current annual production levels in Italy must be $<1 \mathrm{t} /$ year. $^{43}$ (2) One study reported that PFHxS production by one major producer in China began only in 2009 and was then ceased in $2012 ;^{44}$ however, the sources of this information are not referenced in that study.

Using the production volumes of POSF, PHxSF, and PDSF reported by $3 \mathrm{M}$ in 1997 (where PHxSF and PDSF were assumed to be unintentional byproducts), ${ }^{22}$ we scaled down the annual POSF production volumes from 1958 to 2030 developed in our previous study ${ }^{3}$ to estimate annual PHxSF and PDSF production volumes. As the mixture produced by the electrochemical fluorination process may vary from plant to plant due to differences in process conditions, raw materials, and equipment, ${ }^{37}$ we made the following assumptions. (1) For production between 1958 and 2002, almost all production of POSF occurred in CG1, and we used scaling factors of $12 \%$ for PHxSF and $0.6 \%$ for PDSF derived from the $3 \mathrm{M}$ values for 1997..$^{22}$ The scaling factor for PHxSF is also in good agreement with measurements reported in the risk profile of $\mathrm{PFHxS}$ and related substances under the Stockholm Convention. ${ }^{20}$ (2) From 2002 and onward, production of POSF mostly occurred in China and increased after 3M's phase-out in 2002. ${ }^{45}$ For this Chinese production, we applied a scaling factor of $9.3 \%$ to previously estimated POSF production levels in $\mathrm{China}^{3}$ to calculate annual PHxSF production volumes. This factor was derived from the average of three reported impurity fractions of PFHxS in commercial PFOS samples from Chinese manufacturers. $^{42}$ No reported levels of $C_{10}$ impurities in Chinese commercial PFOS or its precursors were found, and 
therefore, we used the same scaling factor of $0.6 \%$ as used in CG1 to represent PDSF production as impurities in POSF produced in China. Detailed annual production estimates are provided in section S2.1 of the Supporting Information.

Quantification of Emissions. In accordance with our earlier study, ${ }^{3}$ emissions of PHxSF, PFHxS, and its major precursors perfluorohexanesulfonyl sulfonamide/-amidoethanols $(x \mathrm{FHxSA} / \mathrm{Es})$, as well as of PDSF, PFDS, and $x \mathrm{FDSA} / \mathrm{Es}$, from the manufacturing, use, and disposal phases were calculated across the lifetimes of products containing relevant substances. Where limited information was available, higherand lower-bound scenarios were applied to the parameters to generate a range of plausible emissions. The following key scenario ranges were applied. (1) Given the large number of potential products and their varying emission rates during use and disposal, lower and higher fractional releases of all substances from consumer use and disposal into the environment were applied ( $10 \%$ vs $100 \%)$. (2) As polymeric precursors have degradation half-lives that are much longer than those of nonpolymeric precursors, they were separately considered in the calculations. Given the uncertain mix of nonpolymeric versus polymeric precursors manufactured in CG1, two scenario values of estimated polymeric-to-nonpolymeric ratios were considered (20\% polymers vs $80 \%$ polymers). (3) Given the uncertain degradation half-lives of polymeric products, lower and higher scenario degradation half-lives were considered (100 years vs 10 years). A detailed description of the specific parameters used in the calculations of the emissions (e.g., production volumes, manufacturing emission factors, polymeric ratios, and homologue compositions) is included in section S2 of the Supporting Information. The developed $\mathrm{C}_{6}$ emission inventory also considered relevant emissions of $\mathrm{C}_{6}$ impurities from the developed $\mathrm{C}_{10}$ inventory and vice versa.

Modeling and Comparison with Field Measurements. The resulting emission inventories were input into the CliMoChem model, which divides the Earth into 10 latitudinal zones of $18^{\circ}$ width and contains environmental compartments of tropospheric air, bare and vegetation-covered soil, vegetation, oceanic surface water, snow, and ice. Details of the model's structure and processes were provided in previous studies. ${ }^{46-48}$ The model was run separately for the $C_{6}$ and $C_{10}$ substances at a monthly resolution from 1958 until 2030. The estimated yearly emissions of PHxSF, PDSF, PFHxS, PFDS, $x \mathrm{FHxSA} / \mathrm{Es}$, and $x \mathrm{FDSA} / \mathrm{Es}$ were equally distributed over each year and assigned to latitudinal zones based on reported ${ }^{45}$ and estimated geographical distributions of production, use, and disposal. Partition coefficients as well as degradation pathways, rate constants, and yields in individual compartments were defined in the model largely based on values reported for the corresponding $\mathrm{C}_{8}$ homologues of the substances because only limited studies ${ }^{49,50}$ of these parameters for the $C_{6}$ and $C_{10}$ homologues were available. All details about the input parameters and modeling scenarios applied are provided in section S3 of the Supporting Information.

The modeled environmental concentrations in ocean water were compared with reported field concentrations in oceanic surface water for PFHxS ${ }^{51-60}$ and PFDS. ${ }^{56}$ Comparable field concentrations in other environmental compartments were not available. As the CliMoChem model estimates average concentrations within a latitudinal zone, it can be expected that model results are similar to measured concentrations at background sites far from source regions. ${ }^{61}$ Therefore, only reported measurements from field sites representing background concentrations were considered for comparison. Results from sites on industrialized land, surrounded by industrialized land, or near likely sources were not compared with model results. For example, there are measurement results available for, e.g., groundwater, ${ }^{62}$ surface water, ${ }^{63}$ and soil; ${ }^{64}$ however, such local contamination cannot be captured in the CliMoChem model because of its different geographical resolution. Field data that reflect "not detected" or "not quantifiable" concentrations of PFHxS and PFDS were not included for comparison.

\section{RESULTS AND DISCUSSION}

Estimated Global Sum Emissions from PHxSF- and PDSF-Based Products. The estimated emission inventories are summarized in Table 1, including the estimated range of

Table 1. Ranges of Global Estimated Emissions of PFHxS and PHxSF from the Life Cycle of PHxSF-Based Products and of PFDS and PDSF from PDSF-Based Products (rounded to the nearest metric ton)

\begin{tabular}{|c|c|c|c|c|}
\hline & $\begin{array}{c}1958-2002 \\
(t)\end{array}$ & $\begin{array}{c}2003-2015 \\
(t)\end{array}$ & $\begin{array}{c}2016-2030 \\
(t)\end{array}$ & total $(t)$ \\
\hline \multicolumn{5}{|l|}{ PFHxS } \\
\hline $\begin{array}{l}\text { (1) emissions from } \\
\text { production }^{a}\end{array}$ & $86-86$ & $2-2$ & $0-1$ & $88-89$ \\
\hline $\begin{array}{l}\text { (2) emissions from } \\
\text { use and disposal }\end{array}$ & $6-307$ & $3-30$ & $1-21$ & $10-358$ \\
\hline $\begin{array}{l}\text { (3) emissions from } \\
\text { degradation of } \\
x \mathrm{FHxSA} / \mathrm{Es}^{b}\end{array}$ & $1-410$ & $1-124$ & $1-66$ & $3-600$ \\
\hline $\begin{array}{l}\text { (4) emissions from } \\
\text { degradation of } \\
\text { PHxSF }^{b}\end{array}$ & $20-61$ & $1-2$ & $0-1$ & $21-64$ \\
\hline total & $113-864$ & $7-158$ & $2-89$ & $122-1111$ \\
\hline \multicolumn{5}{|l|}{ PFDS } \\
\hline $\begin{array}{l}\text { (1) emissions from } \\
\text { production }^{a}\end{array}$ & $4-5$ & 0 to $<1$ & 0 to $<1$ & $4-5$ \\
\hline $\begin{array}{l}\text { (2) emissions from } \\
\text { use and disposal }\end{array}$ & $33-358$ & $0-6$ & $0-1$ & $33-365$ \\
\hline $\begin{array}{l}\text { (3) emissions from } \\
\text { degradation of } \\
x \text { FDSA/Es }{ }^{b}\end{array}$ & $0-3$ & $0-2$ & $0-1$ & $0-6$ \\
\hline $\begin{array}{l}\text { (4) emissions from } \\
\text { degradation of } \\
\text { PDSF }^{b}\end{array}$ & $1-4$ & 0 to $<1$ & 0 to $<1$ & $1-4$ \\
\hline total & $38-370$ & $0-8$ & $0-2$ & $38-380$ \\
\hline \multicolumn{5}{|l|}{ PHxSF } \\
\hline $\begin{array}{l}\text { (1) emissions from } \\
\text { production }^{a}\end{array}$ & $78-78$ & $2-2$ & $0-1$ & $80-81$ \\
\hline \multicolumn{5}{|l|}{ PDSF } \\
\hline $\begin{array}{l}\text { (1) emissions from } \\
\text { production }^{a}\end{array}$ & $4-4$ & 0 to $<1$ & 0 to $<1$ & $4-4$ \\
\hline
\end{tabular}

${ }^{a}$ Ranges of calculated emissions from production are often small because of the use of single emission factors and production volumes as well as the small uncertainty range of the homologue composition of products (see sections S2.1.2 and S2.1.4 of the Supporting Information). ${ }^{b}$ These values were estimated by the degradation processes implemented in the CliMoChem model.

global total emissions for PFHxS, PFDS, PHxSF, and PDSF and from individual source categories for three time periods. A majority of the emissions are estimated to have occurred from production within CG1 countries during the period before 3 M's phase-out of POSF-based products, with emissions of $\mathrm{PFHxS}$ predicted to continue from product use and disposal $(1-21 \mathrm{t})$ and degradation of $x \mathrm{FHxSA} /$ Es $(1-66 \mathrm{t})$ between 

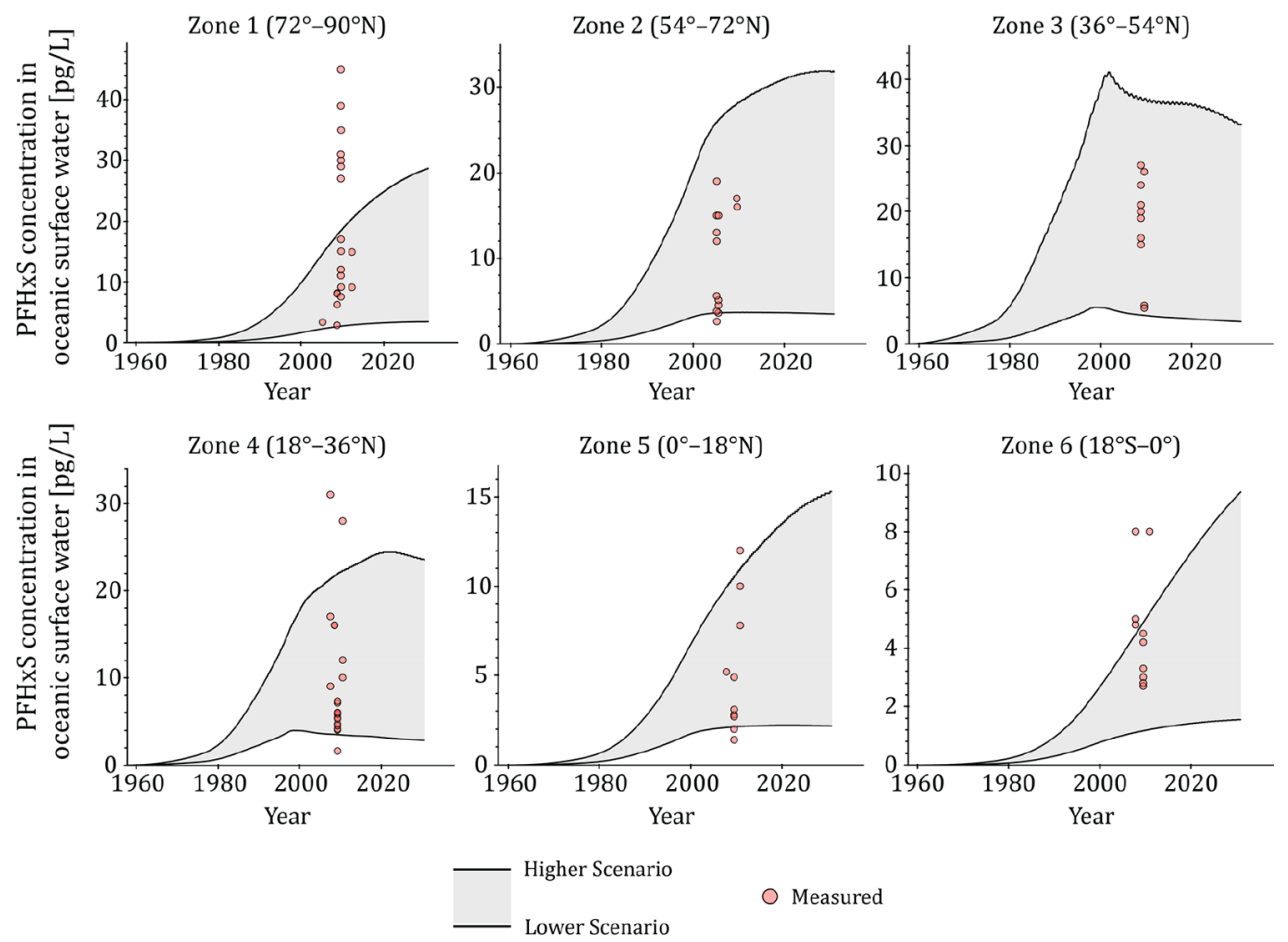

Figure 1. Modeled concentrations (assuming $80 \%$ polymeric compounds in CG1) vs measured field concentrations of PFHxS in oceanic surface water in 1958-2030 in zones 1-6 with different $y$-axis scaling for each. ${ }^{51-60}$ Results for the remaining zones are shown in Figure S8. Figure S9 shows very similar results for modeled concentrations using the scenario of $20 \%$ polymeric compounds in CG1.

2016 and 2030. The unintentional production of PHxSFrelated substances as impurities in Chinese manufacturing of POSF-based products is therefore estimated to be far less significant in comparison. Further emission inventory estimates are available in section S5 of the Supporting Information.

Modeled versus Measured Environmental Concentrations of PFHxS and PFDS. In Figure 1, the modeled environmental background concentrations of $\mathrm{PFHxS}$ in oceanic surface water, derived from the emission inventories assuming $80 \%$ polymeric products in CG1, are compared with field measurements. The model results are plotted for different latitudinal zones with the higher and lower model scenarios shown by the gray band. All results comparing modeled and measured environmental concentrations, including for PFHxS assuming $20 \%$ polymeric products in CG1 as well as for PFDS in oceanic surface water, are provided in section S5.2 of the Supporting Information and show similar trends. The modeled concentrations rise most rapidly in zone 3 , where most production is estimated to occur, and they reach a peak range around 2002 of approximately 5-40 pg/L for PFHxS (5-50 $\mathrm{pg} / \mathrm{L}$ for the $20 \%$ polymeric scenario) and $2-25 \mathrm{pg} / \mathrm{L}$ for PFDS. More northern and southern zones show an increasing delay before concentrations begin to rise after the start of production and in reaching peak concentrations. An increasing delay in these zones can also be seen before modeled concentrations begin to decrease following 3M's phase-out that began in 2000. Such increasing delays are attributed to increasing distances from the emission source zone.

The reported field concentrations generally fit well within the range of modeled concentrations for both PFHxS and PFDS, and all field concentrations are well within the same order of magnitude as the modeled ranges. For $\mathrm{PFHxS},>85 \%$ of the field concentrations are within the modeled range for zones 2-4. For PFDS, three of the five available field measurements are within the modeled range. Resulting model concentration ranges for the 20 and $80 \%$ polymeric scenarios in CG1 are very similar for most zones and result in a maximum peak concentration difference of approximately $20 \%$ $(10 \mathrm{pg} / \mathrm{L})$ for PFHxS in zone 3 and $<1 \%$ for PFDS. The measured field concentrations fit well within resulting modeled concentration ranges from both scenarios.

These results offer strong support that the two emission inventories presented here are plausible and that the hypotheses regarding the historical production volumes of PHxSF- and PDSF-based products are reasonable. The lack of quantitative data on production, uses, disposal, and degradation-related parameters for the substances in the inventory calculations made it necessary to make estimations and assumptions, which have in turn introduced uncertainties into the results. Some of the greatest uncertainties are expected to be within the production and use volumes, the emission factors from the use and disposal phase, and the degradation of polymeric precursors. In addition, there are some known but currently unquantifiable uncertainties, such as potential delays from natural reservoirs such as groundwater and inland surface water. However, many of these uncertainties are expected to have been captured by the lower and higher scenarios of the inventory calculations and modeling parameters. Future studies may help to further narrow the uncertainty range and identify currently existing unknown unknowns. A review of the estimated uncertainties for the applied parameters is provided in section S5.3 of the Supporting Information.

Implications from This Study and Future Perspectives. The modeling results show that elevated environmental concentrations of PHxSF, PDSF, and their derivatives are expected to continue for decades to come, and zones farther from the source regions have not yet even reached their estimated peak concentrations. These estimated trends are 
likely to be widely representative on a large geographical scale; however, the currently nonquantifiable emissions of intentional production in Italy and China of PHxSF-based products may increase levels of local exposure in some areas and cannot be captured by the current study.

Ongoing environmental exposure to these substances has also been discussed in a recent review of trends in concentrations of per- and polyfluoroalkyl substances (PFAS) in various media. ${ }^{30}$ The authors found that PFHxS levels in wildlife have primarily either increasing or inconsistent trends. Trends in PFDS concentrations measured in wildlife were varied or indeterminable. Another recent study was able to reconstruct historical human serum concentrations of PFOA and PFOS after 3M's 2000-2002 phase-out but not that of PFHxS. ${ }^{65}$ This highlights the need for data with a high regional/local resolution to better understand the exposure of certain populations to PFHxS and PFDS.

The results from developing the emissions inventories in this study, as well as from the other recent efforts, ${ }^{30,65}$ underscore a need for a better understanding of the emissions and environmental fate of especially PFHxS precursors. This is notably urgent because these substances have been identified to fulfill the criteria for persistent organic pollutants ${ }^{66}$ and were found to likely lead to significant adverse effects on human health and the environment. ${ }^{32}$ In addition to the need for better, publicly available data on production and uses, samples from ongoing, long-term human and wildlife biomonitoring programs should also be continually analyzed for PFASs in the future to monitor environmental exposure and assess the effectiveness of risk mitigation actions.

This study helps to improve the understanding of the historical production and fate of PHxSF- and PDSF-based compounds. However, even these substances are often considered "legacy" PFASs and have at least some data available enabling the estimation of their environmental emissions. Future efforts should specifically focus on the potentially hundreds to thousands of other, newer PFASs currently on the market with practically no information publicly available describing their production, uses, or releases. ${ }^{67}$ This remains a key, ongoing aspect to be addressed.

\section{ASSOCIATED CONTENT}

\section{S Supporting Information}

The Supporting Information is available free of charge on the ACS Publications website at DOI: 10.1021/acs.estlett.8b00531.

Detailed methodologies for estimating emissions from each source and additional information, figures, and tables (PDF)

\section{AUTHOR INFORMATION}

\section{Corresponding Authors}

*E-mail: justin.boucher@chem.ethz.ch. Phone: +41 4463343 86. Fax: +41446321189.

*E-mail: zhanyun.wang@ifu.baug.ethz.ch. Phone: +41 44633 7066. Fax: +41446331061.

\section{ORCID}

Justin M. Boucher: 0000-0003-0517-7043

Ian T. Cousins: 0000-0002-7035-8660

Martin Scheringer: 0000-0002-0809-7826

Zhanyun Wang: 0000-0001-9914-7659

\section{Notes}

The authors declare no competing financial interest.

\section{ACKNOWLEDGMENTS}

J.M.B. and Z.W. gratefully acknowledge the Swiss Federal Office for the Environment (FOEN) for funding. M.S. gratefully acknowledges financial support by the Czech Ministry of Education, Youth, and Sports (LM2015051) and Masaryk University (CETOCOEN PLUS project, CZ.02.1.01/ 0.0/0.0/15_003/0000469).

\section{REFERENCES}

(1) Paul, A. G.; Jones, K. C.; Sweetman, A. J. A First Global Production, Emission, And Environmental Inventory For Perfluorooctane Sulfonate. Environ. Sci. Technol. 2009, 43, 386-392.

(2) Armitage, J. M.; Schenker, U.; Scheringer, M.; Martin, J. W.; Macleod, M.; Cousins, I. T. Modeling the Global Fate and Transport of Perfluorooctane Sulfonate (PFOS) and Precursor Compounds in Relation to Temporal Trends in Wildlife Exposure. Environ. Sci. Technol. 2009, 43, 9274-9280.

(3) Wang, Z.; Boucher, J. M.; Scheringer, M.; Cousins, I. T.; Hungerbühler, K. Toward a Comprehensive Global Emission Inventory of C4-C10 Perfluoroalkanesulfonic Acids (PFSAs) and Related Precursors: Focus on the Life Cycle of C8-Based Products and Ongoing Industrial Transition. Environ. Sci. Technol. 2017, 51, $4482-4493$.

(4) Löfstedt Gilljam, J.; Leonel, J.; Cousins, I. T.; Benskin, J. P. Is Ongoing Sulfluramid Use in South America a Significant Source of Perfluorooctanesulfonate (PFOS)? Production Inventories, Environmental Fate, and Local Occurrence. Environ. Sci. Technol. 2016, 50, 653-659.

(5) Buser, A.; Morf, L. Substance Flow Analysis for Switzerland: Perfluorinated Surfactants Perfluorooctanesulfonate (PFOS) and Perfluorooctanoic Acid (PFOA). Federal Office for the Environment (FOEN): Bern, Switzerland, 2009.

(6) Place, B. J.; Field, J. A. Identification of Novel Fluorochemicals in Aqueous Film-Forming Foams Used by the US Military. Environ. Sci. Technol. 2012, 46, 7120-7127.

(7) D'Agostino, L. A.; Mabury, S. A. Identification of Novel Fluorinated Surfactants in Aqueous Film Forming Foams and Commercial Surfactant Concentrates. Environ. Sci. Technol. 2014, $48,121-129$.

(8) Barzen-Hanson, K. A.; Roberts, S. C.; Choyke, S.; Oetjen, K.; McAlees, A.; Riddell, N.; McCrindle, R.; Ferguson, P. L.; Higgins, C. P.; Field, J. A. Discovery of 40 Classes of Per- and Polyfluoroalkyl Substances in Historical Aqueous Film-Forming Foams (AFFFs) and AFFF-Impacted Groundwater. Environ. Sci. Technol. 2017, 51, 20472057.

(9) Howell, R. D.; 3M Environmental Laboratory. Environmental Fate and Effects of Fluorochemicals (Presentation). Minnesota Document PTX2695; 1998.

(10) Hubei Hengxin Chemical Co Ltd. Products (HX-601, HX-168) http://www.fluoride-cn.com/product en.html (accessed January 22, 2018).

(11) Dainippon Ink and Chemicals Inc. Prevention of Mist Formation over Plating Baths. JP 54076443, 1979.

(12) Dainippon Ink and Chemicals Inc. N-(Dihydroxypropyl)Perfluoroalkanecarbonamide and -Sulfonamide Derivatives as Antifogging Agents. JP 63208561, 1988.

(13) Minnesota Mining and Manufacturing Co. Mist Suppressant. GB 2077765, 1981.

(14) Hubei Hengxin Chemical Co Ltd. Chromium Fog Inhibitor Tetraethyl Ammonium Perfluorohexanesulfonate and Its Preparation Method. CN 104611733, 2015.

(15) Fraunhofer ITEM. 28 Day Repeated Dermal Contact Study of 3M Test Articles in Sprague-Dawley Rats Final Report Appendix A: Test Sample Re-Analysis (226-1874a) (U.S. EPA Administrative Record 226). 2000. 
(16) Huang, J.; Yu, G.; Mein, S. PFOS in China: Production, Application \& Alternatives (Presentation). http://www.basel.int/ Implementation/POPsWastes/TechnicalGuidelinesarchives/tabid/ 2381/ctl/Download $/ \mathrm{mid} / 13358 /$ Default.aspx?id=13\&ObjID=11613 (accessed January 10, 2018).

(17) Fritztile. Safety Data Sheet FCP-102; 2015.

(18) Fritztile. Safety Data Sheet FCP-300; 2015.

(19) SPIN (Substances in Preparations in Nordic Countries). Substance Record CAS 67584-61-6. http://www.spin2000.net/ spinmyphp/ (accessed January 22, 2018).

(20) Stockholm Convention on Persistent Organic Pollutants. Risk Profile on Perfluorohexane Sulfonic Acid (PFHxS), Its Salts and PFHxS-Related Compounds. 2018.

(21) 3M. Voluntary Use and Exposure Information Profile Perfluorodecane Sulfonate (U.S. EPA Administrative Record 2260579). 2000; pp 56-67.

(22) 3M. Letter to US EPA Re: Phase-out Plan for POSF-Based Products (U.S. EPA Administrative Record 226-0600). 2000; pp 111.

(23) Stockholm Convention on Persistent Organic Pollutants. Technical Paper on the Identification and Assessment of Alternatives to the Use of Perfluorooctane Sulfonic Acid in Open Applications. 2012.

(24) Miteni. Perfluorinated derivatives http://www.miteni.com/ Products/perfluorinatedde.html (accessed February 12, 2018).

(25) Pan, Y.; Zhang, H.; Cui, Q.; Sheng, N.; Yeung, L. W. Y.; Sun, Y.; Guo, Y.; Dai, J. Worldwide Distribution of Novel Perfluoroether Carboxylic and Sulfonic Acids in Surface Water. Environ. Sci. Technol. 2018, 52, 7621-7629.

(26) Gyllenhammar, I.; Berger, U.; Sundström, M.; McCleaf, P.; Eurén, K.; Eriksson, S.; Ahlgren, S.; Lignell, S.; Aune, M.; Kotova, N.; et al. Influence of Contaminated Drinking Water on Perfluoroalkyl Acid Levels in Human Serum - A Case Study from Uppsala, Sweden. Environ. Res. 2015, 140, 673-683.

(27) Rotander, A.; Toms, L.-M. L.; Aylward, L.; Kay, M.; Mueller, J. F. Elevated Levels of PFOS and PFHxS in Firefighters Exposed to Aqueous Film Forming Foam (AFFF). Environ. Int. 2015, 82, 28-34. (28) Jin, C.; Sun, Y.; Islam, A.; Qian, Y.; Ducatman, A. Perfluoroalkyl Acids Including Perfluorooctane Sulfonate and Perfluorohexane Sulfonate in Firefighters. J. Occup. Environ. Med. 2011, 53, 324-328.

(29) Beesoon, S.; Genuis, S. J.; Benskin, J. P.; Martin, J. W. Exceptionally High Serum Concentrations of Perfluorohexanesulfonate in a Canadian. Environ. Sci. Technol. 2012, 46, 12960-12967.

(30) Land, M.; de Wit, C. A.; Cousins, I. T.; Herzke, D.; Johansson, J.; Martin, J. W. What Is the Effect of Phasing out Long-Chain perand Polyfluoroalkyl Substances on the Concentrations of Perfluoroalkyl Acids and Their Precursors in the Environment? A Systematic Review Protocol. Environ. Evid. 2015, 4, 3.

(31) Stockholm Convention on Persistent Organic Pollutants. Proposal to List Perfluorohexane Sulfonic Acid (CAS No: 355-46-4, PFHxS), Its Salts and PFHxS-Related Compounds in Annexes A, B and/or $\mathrm{C}$ to the Stockholm Convention on Persistent Organic Pollutants. 2017.

(32) IISD Reporting Services. 14th Meeting of the Persistent Organic Pollutants Review Committee (POPRC-14) of the Stockholm Convention on Persistent Organic Pollutants: 17-21 September 2018. Earth Negotiation Bulletin 2018, 15, 1-8.

(33) European Chemicals Agency. Inclusion of Substances of Very High Concern in the Candidate List for Eventual Inclusion in Annex XIV - Perfluorohexane-1-Sulphonic Acid and Its Salts (PFHxS). 2017.

(34) Minnesota Mining and Manufacturing Co. Fluorocarbon Sulfonic Acids and Derivatives. US 2732398, 1956.

(35) Kissa, E. Fluorinated Surfactants and Repellents, 2nd ed.; Marcel Dekker, Inc.: New York, 2001.

(36) Siegemund, G.; Schwertfeger, W.; Feiring, A.; Smart, B.; Behr, F.; Vogel, H.; McKusick, B.; Kirsch, P.; Siegemund, G.; Schwertfeger, W.; et al. Fluorine Compounds, Organic. In Ullmann's Encyclopedia of
Industrial Chemistry; Wiley-VCH Verlag GmbH \& Co. KGaA: Weinheim, Germany, 2016; pp 1-56.

(37) 3M. Science of Organic Fluorochemistry. http://www. fluoridealert.org/wp-content/pesticides/pfos.fr.final.docket.0006.pdf (accessed November 8, 2018).

(38) Pearlson, W. H. The Simons Electrochemical Fluorination Process (Commercial Development at 3M). J. Fluorine Chem. 1986, 32, 29-40.

(39) Audenaert, F.; Lens, H.; Rolly, D.; Vander Elst, P. Fluorochemical Textile Repellents-Synthesis and Applications: A 3M Perspective. J. Text. Inst. 1999, 90, 76-94.

(40) Gramstad, T.; Haszeldine, R. N. 512. Perfluoroalkyl Derivatives of Sulphur. Part VI. Perfluoroalkanesulphonic Acids CF3[CF2]NSO3H $(\mathrm{N}=1-7)$. J. Chem. Soc. 1957, 0, 2640-2645.

(41) 3M. Sulfonated Perfluorochemicals in the Environment: Sources, Dispersion, Fate and Effects (AR226-0620) (U.S. EPA Administrative Record 226). 2000.

(42) Jiang, W.; Zhang, Y.; Yang, L.; Chu, X.; Zhu, L. Perfluoroalkyl Acids (PFAAs) with Isomer Analysis in the Commercial PFOS and PFOA Products in China. Chemosphere 2015, 127, 180-187.

(43) European Chemicals Agency. REACH Registered Substances. https://echa.europa.eu/information-on-chemicals/registeredsubstances (accessed September 21, 2018).

(44) Fu, J.; Gao, Y.; Cui, L.; Wang, T.; Liang, Y.; Qu, G.; Yuan, B.; Wang, Y.; Zhang, A.; Jiang, G. Occurrence, Temporal Trends, and Half-Lives of Perfluoroalkyl Acids (PFAAs) in Occupational Workers in China. Sci. Rep. 2016, 6, 38039.

(45) Zhang, L.; Liu, J.; Hu, J.; Liu, C.; Guo, W.; Wang, Q.; Wang, H. The Inventory of Sources, Environmental Releases and Risk Assessment for Perfluorooctane Sulfonate in China. Environ. Pollut. 2012, 165, 193-198.

(46) Scheringer, M.; Wegmann, F.; Fenner, K.; Hungerbühler, K. Investigation of the Cold Condensation of Persistent Organic Pollutants with a Global Multimedia Fate Model. Environ. Sci. Technol. 2000, 34, 1842-1850.

(47) Schenker, U.; Scheringer, M.; Hungerbühler, K. Including Degradation Products of Persistent Organic Pollutants in a Global Multi-Media Box Model. Environ. Sci. Pollut. Res. 2007, 14, 145-152.

(48) Schenker, U.; Scheringer, M.; MacLeod, M.; Martin, J. W.; Cousins, I. T.; Hungerbühler, K. K. Contribution of Volatile Precursor Substances to the Flux of Perfluorooctanoate to the Arctic. Environ. Sci. Technol. 2008, 42, 3710-3716.

(49) Wang, Z.; MacLeod, M.; Cousins, I. T.; Scheringer, M.; Hungerbuehler, K. Using COSMOtherm to Predict Physicochemical Properties of Poly- and Perfluorinated Alkyl Substances (PFASs). Environ. Chem. 2013, 8, 389-398.

(50) Higgins, C. P.; Luthy, R. G. Sorption of Perfluorinated Surfactants on Sediments. Environ. Sci. Technol. 2006, 40, 7251-7256.

(51) Ahrens, L.; Xie, Z.; Ebinghaus, R. Distribution of Perfluoroalkyl Compounds in Seawater from Northern Europe, Atlantic Ocean, and Southern Ocean. Chemosphere 2010, 78, 1011-1016.

(52) Benskin, J. P.; Muir, D. C. G.; Scott, B. F.; Spencer, C.; De Silva, A. O.; Kylin, H.; Martin, J. W.; Morris, A.; Lohmann, R.; Tomy, G.; et al. Perfluoroalkyl Acids in the Atlantic and Canadian Arctic Oceans. Environ. Sci. Technol. 2012, 46, 5815-5823.

(53) Zhao, Z.; Xie, Z.; Möller, A.; Sturm, R.; Tang, J.; Zhang, G.; Ebinghaus, R. Distribution and Long-Range Transport of Polyfluoroalkyl Substances in the Arctic, Atlantic Ocean and Antarctic Coast. Environ. Pollut. 2012, 170, 71-77.

(54) Zhao, Z.; Xie, Z.; Tang, J.; Sturm, R.; Chen, Y.; Zhang, G.; Ebinghaus, R. Seasonal Variations and Spatial Distributions of Perfluoroalkyl Substances in the Rivers Elbe and Lower Weser and the North Sea. Chemosphere 2015, 129, 118-125.

(55) Busch, J.; Ahrens, L.; Xie, Z.; Sturm, R.; Ebinghaus, R. Polyfluoroalkyl Compounds in the East Greenland Arctic Ocean. J. Environ. Monit. 2010, 12, 1242-1246.

(56) Cai, M.; Zhao, Z.; Yin, Z.; Ahrens, L.; Huang, P.; Cai, M.; Yang, H.; He, J.; Sturm, R.; Ebinghaus, R.; et al. Occurrence of 
Perfluoroalkyl Compounds in Surface Waters from the North Pacific to the Arctic Ocean. Environ. Sci. Technol. 2012, 46, 661-668.

(57) Wei, S.; Chen, L. Q.; Taniyasu, S.; So, M. K.; Murphy, M. B.; Yamashita, N.; Yeung, L. W. Y.; Lam, P. K. S. Distribution of Perfluorinated Compounds in Surface Seawaters between Asia and Antarctica. Mar. Pollut. Bull. 2007, 54, 1813-1818.

(58) Ahrens, L.; Barber, J. L.; Xie, Z.; Ebinghaus, R. Longitudinal and Latitudinal Distribution of Perfluoroalkyl Compounds in the Surface Water of the Atlantic Ocean. Environ. Sci. Technol. 2009, 43, 3122-3127.

(59) Yeung, L. W. Y.; Dassuncao, C.; Mabury, S.; Sunderland, E. M.; Zhang, X.; Lohmann, R. Vertical Profiles, Sources, and Transport of PFASs in the Arctic Ocean. Environ. Sci. Technol. 2017, 51, 67356744.

(60) Casal, P.; Zhang, Y.; Martin, J. W.; Pizarro, M.; Jiménez, B.; Dachs, J. Role of Snow Deposition of Perfluoroalkylated Substances at Coastal Livingston Island (Maritime Antarctica). Environ. Sci. Technol. 2017, 51, 8460-8470.

(61) Becker, L.; Scheringer, M.; Schenker, U.; Hungerbühler, K. Assessment of the Environmental Persistence and Long-Range Transport of Endosulfan. Environ. Pollut. 2011, 159, 1737-1743.

(62) Anderson, R. H.; Long, G. C.; Porter, R. C.; Anderson, J. K. Occurrence of Select Perfluoroalkyl Substances at U.S. Air Force Aqueous Film-Forming Foam Release Sites Other than Fire-Training Areas: Field-Validation of Critical Fate and Transport Properties. Chemosphere 2016, 150, 678-685.

(63) Chen, M.; Wang, Q.; Shan, G.; Zhu, L.; Yang, L.; Liu, M. Occurrence, Partitioning and Bioaccumulation of Emerging and Legacy per- and Polyfluoroalkyl Substances in Taihu Lake, China. Sci. Total Environ. 2018, 634, 251-259.

(64) Rankin, K.; Mabury, S. A.; Jenkins, T. M.; Washington, J. W. A North American and Global Survey of Perfluoroalkyl Substances in Surface Soils: Distribution Patterns and Mode of Occurrence. Chemosphere 2016, 161, 333-341.

(65) Gomis, M. I.; Vestergren, R.; MacLeod, M.; Mueller, J. F.; Cousins, I. T. Historical Human Exposure to Perfluoroalkyl Acids in the United States and Australia Reconstructed from Biomonitoring Data Using Population-Based Pharmacokinetic Modelling. Environ. Int. 2017, 108, 92-102.

(66) Stockholm Convention on Persistent Organic Pollutants. Report of the Persistent Organic Pollutants Review Committee on the Work of Its Thirteenth Meeting. 2017.

(67) Wang, Z.; DeWitt, J. C.; Higgins, C. P.; Cousins, I. T. A NeverEnding Story of Per- and Polyfluoroalkyl Substances (PFASs)? Environ. Sci. Technol. 2017, 51, 2508-2518. 